\title{
Recent results from the study of emittance evolution in MICE
}

Hunt, C.**

Imperial College London,

E-Mail: christopher.hunt08@imperial.ac.uk

The Muon Ionization Cooling Experiment (MICE) has measured the change in emittance due to ionization energy loss. Large aperture solenoid magnets were used to focus muons through Lithium-hydride, liquid hydrogen and empty absorbers. Diagnostic devices were placed upstream and downstream of the absorber, enabling the phase-space coordinates of individual muons to be reconstructed. By observing the properties of ensembles of muons, the evolution of the beam emittance was studied. The phase-space was analysed using single particle amplitude distributions and shows a clear increase in the core density of the beam, a direct effect of ionization cooling.

The 20th International Workshop on Neutrinos (NuFact2018)

12-18 August 2018

Blacksburg, Virginia

* Speaker.

${ }^{\dagger}$ on behalf of the MICE collaboration 


\section{Muon Ionization Cooling Experiment}

The International Muon Ionization Cooling Experiment (MICE) [1], aims to demonstrate ionization cooling so that it may be exploited in future cooling-channel designs [2, 3, 4]. The MICE experiment produces pions using a titanium target, dipped into the beam halo of the $800 \mathrm{MeV}$ ISIS proton synchrotron [5]. The emitted pions are captured and allowed to decay, such that the produced muons may by focussed and transported to the cooling channel. Twelve superconducting coils arranged in 3 cryostats were used to transport the beam through a central absorber chamber. Three different absorbers were used: liquid hydrogen, lithium hydride ( $\mathrm{LiH})$ and a plastic wedgeshaped absorber. Performance of the wedge-shaped absorber is not reported in this paper. The upstream and downstream ensembles were reconstructed particle-by-particle using a scintillating fibre tracker [6,7], such that the effect of the absorber could be investigated.

\section{Ionization Cooling}

Ionization cooling that involves focussing the beam through a "cooling" material (the absorber), in order to reduce normalised transverse emittance. The absorber material isotropically reduces the beam momentum through the process of ionization energy losses. Low- $\mathrm{Z}$ materials and strong focussing fields are favoured in order to mitigate the effects of multiple Coulomb scattering.

The cooling equation (eq. 2.1) describes the evolution of normalised transverse emittance, $\varepsilon_{\perp}$, of a beam with betatron function, $\beta_{\perp}$, and energy, $E$, through a material with radiation length, $X_{0}$,

$$
\frac{d \varepsilon_{\perp}}{d x}=-\frac{\varepsilon_{\perp}}{\beta^{2} E}\left\langle\frac{d E}{d x}\right\rangle+\frac{\beta_{\perp}(13.6 \mathrm{MeV} / \mathrm{c})^{2}}{2 \beta^{3} E m_{\mu} X_{0}}
$$

where $m_{\mu}$ is the muon mass, $\beta$ is the relativistic velocity of the beam and $x$ is the thickness of the absorber. In order to understand the evolution of the beam on a particle-by-particle basis, single particle amplitudes were calculated to demonstrate the migration of muons into (cooling) and out of (heating) the beam core. An amplitude, A, is calculated as, $A=\varepsilon_{\perp} \mathbf{x} \boldsymbol{\Sigma}^{-1} \mathbf{x}$, where $\mathbf{x}=\left(x, p_{x}, y, p_{y}\right)$ is the individual muon phase-space vector relative to the beam centroid, and $\boldsymbol{\Sigma}$ is the covariance matrix of the current ensemble, with RMS emittance, $\varepsilon_{\perp}$. In order to prevent tail events from biasing the orientation of the core, events with larger amplitudes are iteratively removed from the calculation of the covariance matrix.

\section{Particle Selection}

Each event was required to contain precisely one muon throughout the entire experiment. Events with additional reconstructed tracks were rejected. Each muon was then required to pass a Time-of-Flight (ToF) [8] cut on entry to the cooling channel and momentum selection (135$145 \mathrm{MeV} / \mathrm{c}$ ) within the upstream tracker. The momentum and ToF measurements were required to be consistent with the muon mass. Goodness of fit tests ensure that each track was well fitted upstream and downstream, reducing the possibility of noise contamination and misidentification. 
A loose momentum selection was applied to the downstream tracker to ensure that poorly reconstructed tracks were removed. The event selection was primarily performed on the upstream sample. Cuts applied to the downstream sample were only made to ensure that the transmitted muons were well reconstructed. This ensures that we do not preferentially select muons that have migrated towards the beam core.

\section{Results}

The upstream and downstream samples were analysed independently. The amplitude distributions for each sample were calculated and summed such that the ratio of the cumulative distributions could be calculated. This is shown in figure 1 for the three absorber settings and two nominal emittance settings. The excess in the lowest bins demonstrates the migration of particles towards lower amplitudes, which is only achieved if a fraction of the beam has been cooled. Additionally the lowest transmission is noted to be $94 \%$ in the $10 \mathrm{~mm}$ settings, as seen as the value in the final bin. A Monte Carlo simulation was developed which reproduces the performance of the experiment to

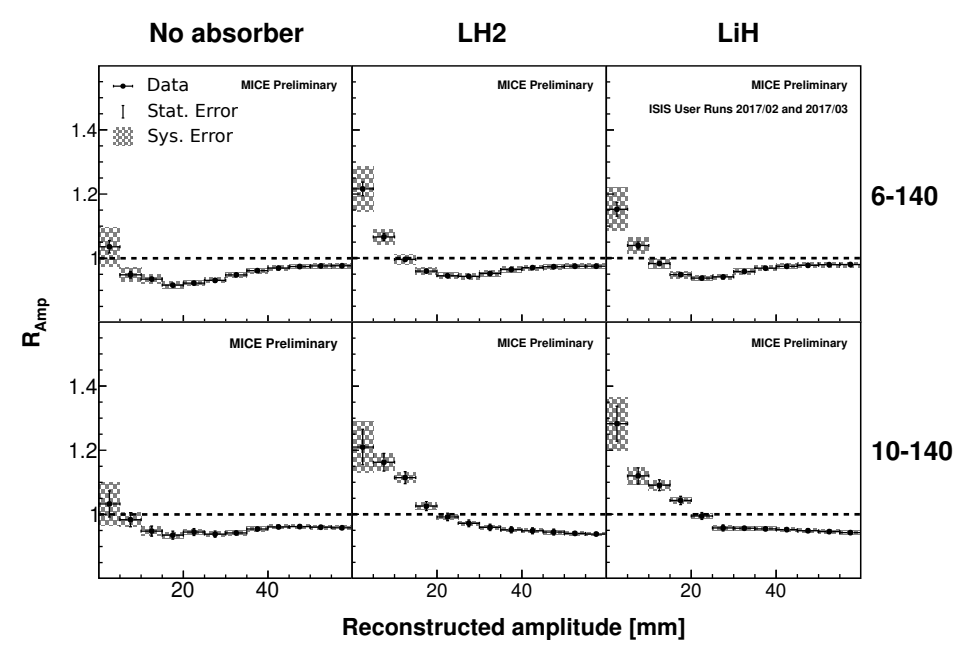

Figure 1: The ratio between the upstream and downstream cumulative amplitude distributions.

high precision. This was used to estimate the systematic uncertainties by varying the known parameters and evaluating the effect on the final amplitude distributions. Systematic corrections were calculated for each bin in amplitude to account for the evaluated residuals. The uncertainty of the correction was included as the systematic error bar.

\section{Conclusion}

Data were taken within the MICE cooling channel in order to reconstruct the transverse phasespace upstream and downstream of an absorber. The migration in single particle amplitudes can be seen to demonstrate a clear increase in the core density, a signal which is only present due to ionization cooling. This is seen in two materials, $\mathrm{LiH}$ and liquid hydrogen, with the empty absorber producing no statistically significant change. Systematic uncertainties have been calculated for each bin in amplitude using a Monte Carlo simulation that is in good agreement with the reconstructed data. 


\section{References}

[1] Muon Ionisation Cooling Experiment. http://www.mice.iit.edu/.

[2] S. Geer, Neutrino beams from muon storage rings: Characteristics and physics potential, Phys. Rev. D 57 (1998) 6989.

[3] M. Apollonio et al., Oscillation Physics with a Neutrino Factory, hep-ph / 0210192.

[4] T. I. A. W. Group, Accelerator design concept for future neutrino facilities, Journal of Instrumentation 4 (2009) P07001.

[5] D. J. S. Findlay, Isis - pulsed neutron and muon source, in 2007 IEEE Particle Accelerator Conference (PAC), pp. 695-699, June, 2007, DOI.

[6] M. Ellis, P. Hobson, P. Kyberd, J. Nebrensky, A. Bross, J. Fagan et al., The design, construction and performance of the $\{M I C E\}$ scintillating fibre trackers, Nuclear Instruments and Methods in Physics Research Section A: Accelerators, Spectrometers, Detectors and Associated Equipment 659 (2011) 136

[7] A. Dobbs, C. Hunt, K. Long, E. Santos, M. A. Uchida, P. Kyberd et al., The reconstruction software for the MICE scintillating fibre trackers, JINST 11 (2016) T12001 [1610.05161].

[8] R. Bertoni et al., The design and commissioning of the MICE upstream time-of-flight system, Nuclear Instruments and Methods in Physics Research Section A: Accelerators, Spectrometers, Detectors and Associated Equipment 615 (2010) 14 . 